\title{
The Effect of Modified Audit Opinion on Debt Contracts
}

\author{
Naufal Zaky ${ }^{1}$ and Sylvia Veronica Siregar ${ }^{2}$ \\ ${ }^{1,2}$ Accounting Department, Faculty of Economy and Business University Indonesia, Indonesia \\ 1'naufalzakyz@gmail.com, ${ }^{2}$ sylvia.veronica@ui.ac.id
}

\begin{abstract}
The purpose of this study is to examine the effect of a modified audit opinion on the debt contract (interest spread, loan size, covenant, collateral, and loan maturity). Samples of this study are credit facilities obtained by listed firms in 2013-2016 with total observations of 660. Results of this study show that in general there is limited effect of modified audit opinion on debt contract. We do not find type of modified audit opinion causes higher interest rates. Modified audit opinion has significant effect on reducing the number of financial covenants. We only find evidence that material uncertainties type of modified audit opinion causes higher general covenant. We also only find evidence of the effect of modified audit opinion on loan maturity for going concern opinion type. Modified audit opinion does not affect loan size and the possibility of the need collateral in the debt contract. The insignificant results of modified audit opinion on debt contracts maybe due to lenders in Indonesia still use soft information (relationship lending) rather than hard information.
\end{abstract}

Keywords. Covenant, Debt Contract, Interest Spread, Loan Maturity, Modified Audit Opinion

\section{Introduction}

An audit report is the auditor's primary way of communicating with users of financial statements, of information found by the auditor when conducting the audit process[1].In the process of assessing whether the financial statements have been presented fairly, the auditor has an important role which reflected in the form of an audit opinion.

In this study, audit opinion is divided into two, namely the unmodified audit opinion that the financial statements have been presented fairly and in accordance with existing accounting standards and modified audit opinion which consists of unqualified opinion with explanatory paragraph, qualified opinion, adverse opinion, and disclaimer opinion. The difference between unmodified audit opinion and modified audit opinion is that in the modified audit opinion there is a paragraph explaining why giving such opinion which is inconsistency in the use of accounting standards, the emphasis of material uncertainty, and opinion on the going concern[2].

Several previous studies[3] on modified audit opinion focus on market reaction to those opinions associated with equity investors. However, modified audit opinion also uses information from financial reports and audit reports. Financial institutions every day lend money to companies taking into account information from financial statements [4]. In that case the lenders become the primary financial statement user whose credibility is affected by the audit report, this is because lenders use financial statements as major factor in the credit decision [5]. 
According to Chen[2] the information in the financial statements is usually used in debt contracts. Contract terms often use a lot of accounting numbers in the financial statements that are used to monitor the performance of the company. Lenders should react with a modified audit opinion in determining and monitoring the provision of credit as the audit opinion provide additional information about the company's financial condition [2].

Based on this background, this research will examine the effect of modified audit opinion on debt contracts. Debt contracts examined in this study consist of interest rates, loan size, covenant, collateral, and loan term. Modified audit opinion will be further grouped into two major groups that are caused by inconsistency and inadequacy. Inconsistency then also subdivided into two that are caused by changes in accounting standards and restatements. Inadequacy is divided into material uncertainty and going concern. This study conducts similar study with that of Chen [2] use listed companies in USA as samples, and this study examine listed firms in Indonesia. In USA where the characteristics is market-based financial system, Chen [2] find significant effect of modified audit opinion on debt contracts. Whereas in Indonesia has different characteristics, which is bank-based financial systems. Therefore it is interesting to examine whether the results also hold in market with bank-based financial systems. There is a study in Indonesia examining the effect of modified audit opinion on debt contract [6]. The difference is that this research not only examine loan size but also other contracts terms that is interest rate, covenant, collateral, and loan term. In addition, type of modified audit opinion used are also grouped into several types as explained before.

\section{Literature Review and Hypotheses Development}

Lenders incorporate covenants in the debt contracts to limit management decisions that will harm the lender [7].When associated with monitoring, [8] suggest that debt financing can improve the monitoring mechanism that will reduce agency costs within the company. This is because monitoring is also done by the lender. Jensen and [7] suggest that a growing number of debt contracts that rely on accounting numbers will lead to demand for higher quality audits.

The audit report is a key way for the auditor to convey their degree of certainty, that the financial statements have reflected the company's economic activity[2]. Broadly speaking, audit opinion can be grouped into two types, namely unmodified audit opinion and modified audit opinion. In this study modified audit opinion is grouped into four types, that is, unqualified opinion with explanatory paragraph, qualified opinion, adverse opinion, and disclaimer.

Modified audit opinion then is divided into two: inconsistency and inadequacy. Opinion on inconsistencies reflects the difficulty of comparing the company's financial statements annually, which is one of the considerations for lenders. This is in accordance with Kent and Munro[9],the main thing that the lender is concerned about is reliable, useful and comparable information. Inconsistencies are then subdivided into two, which are caused by changes in the use of accounting principles and restatements. Changes in the use of accounting principles affect the comparability of financial statements. The restatement reflects that the information possessed by the previous lenders becomes inaccurate, making it necessary to do a risk assessment again[10]. Some restatements in financial statements relate to complex transactions or deliberate manipulation [11] The restatement also provides a question of the credibility of the company's financial statements in the future [12]. According to Chen[2] although information relating to changes in the use of accounting principles and restatements is contained in the notes to the financial statements, the auditors have their own considerations as to why it should be presented in the audit report. This happens because not all changes in the use of accounting principles or restatements on the notes to the financial statements will be reported in the audit report. Opinion about inadequacy is subdivided into two components: going concern and material uncertainty. 
Opinions about going concern reflect that key assumptions in an accounting model have been violated while material uncertainties illustrate future unknown economic decisions[2].

The purpose of the use of debt covenant is to tighten decision-making and investments that reduce the value of claims of debtors (DeFond and Jiambalvo, 1994). [13]Ball, Bushman, and Vasvari divides covenant into financial covenants and general covenants. Financial covenants identify the set ratios and figures contained in the financial statements. The general covenant usually deals with the activities of the company, such as tightened dividend payout policy and investment decisions [14].

There are several studies examining the association of audit opinion on debt contracts. Firth[15] find that audit opinion related to going concern and asset valuation issues significantly affecting the decision in lending. Bamber and Stratton[16] show that when a company has a modified audit opinion, the loan officer will perform a more stringent risk assessment and demand a higher interest rate. In addition, the possibility to grant loans also decreased.

Graham, Li and Qiu[10], find that compared to the loans obtained before the restatement, the loans obtained after the restatement have a higher loan spreads, shorter loan terms, and more stringent terms of agreement. Sormunen [17] examines the usefulness of qualified audit opinion of small and medium entrepreneurs on lending decisions. The result shows that bank employees assume that the qualified audit reports have information, but in their use it is not the main input for decision making.

Another study by Gomez-Guillamon [18] concludes that audit reports play an important role in decision-making for investment as well as lending. In addition, audit reports also affect the amount of money provided for investment and loans. Guiral-Contreras [4] show that qualified audit report is a useful evidence for loan officers. They also concluded that the audit report is used as a warning sign for loan officers to be more critical in making loan decisions.

There are also studies that find different results. Lin, Jiang, and Xu [19] find that modified audit opinion has no effect to the loan size in 1998 until 2004, however modified audit opinion began to have significant influence in 2005 and 2006. Niemi and Sundgren[20] find that modified audit opinion has no effect on loan size.

Chen [2] examine the effect of audit opinion on the debt contract (interest spreads, loan size, covenants, collateral) in the United States. The results show that compared to loans obtained after unqualified audit opinion, loans obtained after a modified audit opinion have a relationship with higher interest spreads, less financial covenants, more general covenants, less loan size, and more needs of collateral.

In Indonesia, [6] Cahyaningrum and Fitriany find that audit opinion does not have an economic impact on the company because it is not used by banks and financial companies in lending decisions unless a company gets unqualified opinion with an explanation paragraph concerning going concern and disclaimer.

According to Blackwell [21] companies that have been through the audit process have a lower interest rate than the company that has not been audited. This is because the audited financial statements have better quality. If a modified audit opinion communicates the quality of a company's financial statements or credit risk, the lender should respond by providing a higher interest rate to reflect a higher credit risk.

H1: New loan obtained after modified audit opinion is issued have a higher loan spreads than new loans obtained after unqualified opinion is issued

Accounting information and ratio information in financial statements are widely used as a means of monitoring the performance of borrowers. This can be done if there is certainty that the financial information reflects the actual performance of the company. If the figures in the financial statements are not considered reliable then the borrower may use more general 
covenants governing the activities of the borrower[2] .Moreover, according to Czerney[22], if the opinion of inconsistency is an indicator to have the possibility of subsequent misstatement, then the financial covenant cannot be used as a tool to monitor the borrower.

H2: New loan obtained after modified audit opinion is issued have a lower financial covenants and higher general covenants compared to new loans after unqualified opinion is issued

If the audit report indicates a risk in the company, then the borrower will provide a lower loan amount, requires collateral, and shorter loan term [2] .

H3: New loan obtained after modified audit opinion have lower loan size, require collateral, and shorter loan term compared to new loans after unqualified opinion issued.

\section{Research Method}

The research model used in this study is based on Chen et al. (2016):

InterestRate $=\alpha+\beta_{1} M A O+\beta_{2}$ AFTERMAO $+\beta_{3}$ FINCOV $+\beta_{4} L O A N S I Z E+\beta_{5}$ MTRTY

$+\beta_{6}$ COLLATERAL $+\beta_{7}$ NUMLDRS $+\beta_{8}$ INSTITOR $+\beta_{9} R E V O L V E R$

$+\beta_{10}$ SIZE $+\beta_{11} L E V+\beta_{12}$ PROF $+\beta_{13}$ TANGIBILITY $+\beta_{14}$ MTB

FinancialCovenant$$
+\beta_{15} A B A C R+\beta_{16} C F V O L+\beta_{17} D 2014+\beta_{18} D 2015+\beta_{19} D 2016+\varepsilon
$$

$$
\begin{aligned}
& =\alpha+\beta_{1} M A O+\beta_{2} \text { AFTERMAO }+\beta_{3} \text { INTRST }+\beta_{4} \text { LOANSIZE } \\
& +\beta_{5} \text { MTRTY }+\beta_{6} \text { COLLATERAL }+\beta_{7} \text { NUMLDRS }+\beta_{8} \text { INSTITOR } \\
& +\beta_{9} \text { REVOLVER }+\beta_{10} \text { SIZE }+\beta_{11} L E V+\beta_{12} \text { PROF }+\beta_{13} \text { TANGIBILITY } \\
& +\beta_{14} M T B+\beta_{15} A B A C R+\beta_{16} C F V O L+\beta_{17} D 2014+\beta_{18} D 2015 \\
& \text { GeneralCovenant } \\
& +\beta_{19} D 2016+\varepsilon \\
& =\alpha+\beta_{1} M A O+\beta_{2} \text { AFTERMAO }+\beta_{3} \text { INTRST }+\beta_{4} L O A N S I Z E \\
& +\beta_{5} \text { MTRTY }+\beta_{6} \text { COLLATERAL }+\beta_{7} \text { NUMLDRS }+\beta_{8} \text { INSTITOR } \\
& +\beta_{9} \text { REVOLVER }+\beta_{10} \text { SIZE }+\beta_{11} L E V+\beta_{12} \text { PROF }+\beta_{13} \text { TANGIBILITY } \\
& +\beta_{14} M T B+\beta_{15} A B A C R+\beta_{16} C F V O L+\beta_{17} D 2014+\beta_{18} D 2015 \\
& +\beta_{19} D 2016+\varepsilon \\
& \text { LoanSize }=\alpha+\beta_{1} M A O+\beta_{2} \text { AFTERMAO }+\beta_{3} \text { INTRST }+\beta_{4} \text { FINCOV }+\beta_{5} \text { MTRTY } \\
& +\beta_{6} \text { COLLATERAL }+\beta_{7} \text { NUMLDRS }+\beta_{8} \text { INSTITOR }+\beta_{9} \text { REVOLVER } \\
& +\beta_{10} \text { SIZE }+\beta_{11} L E V+\beta_{12} \text { PROF }+\beta_{13} \text { TANGIBILITY }+\beta_{14} \text { MTB } \\
& +\beta_{15} A B A C R+\beta_{16} C F V O L+\beta_{17} D 2014+\beta_{18} D 2015+\beta_{19} D 2016+\varepsilon \\
& \text { Maturity }=\alpha+\beta_{1} \text { MAO }+\beta_{2} \text { AFTERMAO }+\beta_{3} \text { INTRST }+\beta_{4} \text { LOANSIZE }+\beta_{5} \text { FINCOV } \\
& +\beta_{6} \text { COLLATERAL }+\beta_{7} \text { NUMLDRS }+\beta_{8} \text { INSTITOR }+\beta_{9} \text { REVOLVER } \\
& +\beta_{10} \text { SIZE }+\beta_{11} L E V+\beta_{12} \text { PROF }+\beta_{13} \text { TANGIBILITY }+\beta_{14} \text { MTB } \\
& +\beta_{15} A B A C R+\beta_{16} C F V O L+\beta_{17} D 2014+\beta_{18} D 2015+\beta_{19} D 2016+\varepsilon
\end{aligned}
$$

Loan Terms represents a specific debt covenants, i.e. interest spread, number of financial covenants, number of general covenants, loan size, collateral, and maturity. Interest spread is the interest rate of each credit facility less the LIBOR rate. The company is said to have a financial covenant if the company must comply with the requirements relating to the company's financial ratios in each credit facility. If the credit facility has financial covenant and not explained the amount will be used financial covenant from other credit facilities with the same lender. If the credit facility has financial covenant and not explained the amount and no other credit facilities from the same lender will be assessed by having a financial covenant. The company is said to have a general covenant if it has the requirements to be followed which regulate the economic activities undertaken by the company. If the credit facility has a general 
covenant and no information is available, the general covenant of another credit facility with the same lender will be used. If the credit facility has a general covenant and not explained the disclosure and no other credit facilities from the same lender, then it will be assessed as having a general covenant. Collateral is an indicator variable related to whether the credit facility requires a collateral, 1 if the loan requires collateral and 0 if otherwise. Maturity is the time span of the loan received until the due date of loan repayment. Loan size is calculated with natural logarithm of loan size.

MAO is an indicator variable of modified audit opinion where 1 if the company gets a modified audit opinion and 0 if otherwise. AfterMAO is an indicator variable of 1 if the company currently receives an unqualified opinion, but gets a modified audit opinion at least once in the previous three years and 0 if otherwise.

In addition, this study also examines the effect of type of MAO on the debt contract.MAO is group into Inconsistency ( 1 if described in the audit report that there is accounting change or restatement and 0 if otherwise), Inadequacy ( 1 if described in the audit report that there Material uncertainty or Going Concern issue and 0 if otherwise).

There are several control variables included in the research model.

Number of lenders (NUMLDRS) in the syndicate loan is included because more parties participating in syndicate loan the higher the quality of the borrower [2] . Institutional Investor (INSTITOR) is an indicator variable ( 1 if credit facilities obtained from non-financial institutions and 1 if otherwise). REVOLVER is an indicator variable related to credit facilities received by companies of revolving type. SIZE represents company size, as smaller companies tend to have higher default risk. LOANSIZE is also included for loan spread model because larger loans tend to have a smaller interest rate. Financial leverage (LEV) is Long term debt divided by total assets. PROF is measured from EBITDA divided by total assets. This is included because usually companies with high profitability will have a low risk of default and can get lower spread. TANGIBILITY is tangible assets divided by the total a set. MTB are the market capitalization divided by total equity. ABACR is the absolute value of discretionary accruals calculated using the Jones model[23]. CFVOL is the standard deviations from quarterly cash flow from operating activities divided by total assets.

This study uses a sample of new loans obtained by nonfinancial sector companies in the period 2013 to 2016. Sample selection criteria are as follow: (1) The company is listed on BEI in 2013 to 2016; (2) Nonfinancial institutions; (3) has audited financial statements from 2013 to 2016; (4) received modified audit opinion at least once in 2013 until 2016; (5) obtained credit facilities in 2013 to 2016; (6) Credit facility information is described in the notes to the financial statements. Based on sampling criteria, we have 660 total observations as shown in Table 1.

\section{Results}

Table 2 shows descriptive statistics of variables. Based on Table2, the average interest rate on the credit facility is 0.0822 . The credit facility having an amount of general covenants maximum of 27 and financial covenants maximum of 6 . Around $47.27 \%$ received modified audit opinion in the previous year before new loan. The proportion of MAO in this study is greater than that of Chen et al. (2016), which has a proportion of 37.8\% MAO. Modified type of audit opinion that is most is ACCTCHG of $28.64 \%$. While the least type of modified audit opinion is material uncertainty has a frequency of $2.88 \%$ of the 660 observations. The regression results are presented in Table 3 - Table 8. From those tables, MAO only has significant effect on financial covenant other dependent variable is not significant. It is different with the results of Chen [2]. However, these results are consistent with Houghton[24] and Miller and Smith[25] that the results of audit have no economic impact on the debt contract. Johnson [26] , also find that audit 
report does not affect lending decisions. Carter (1984) also finds that audit services does not affect the interest rate lending.

This results maybe because some of the information described in an explanatory paragraph has been described in the notes to the financial statements and lenders use other data such as the characteristics of the loan and the characteristics of the company. Houghton[24] also explains that information from audit report is not able to compete with that from e other resources. In this study the characteristics of the loan and companies are also included in the testing in control variables and most have a significant effect.

Variable INCONSISTENCY affect negatively for general covenants and interest rate in the negative. The same results also hold for ACCTCHG and RESTATEMENT variables that also affect general covenants negatively.

Table 1. Sample Selection

Criteria Observations

Listed in Indonesia Stock Exchange in 2017

Financial institutions

Listed after 31 December 2014

Did not received MAOs during the study period

Do not have and/or do not explain about credit facility in notes to the financial statements

Incomplete financial statements information

Total firms

Total firm-years

Number of credit facilities

Number of credit facilities without information on covenant

Table 2. Descriptive Statistics

Panel A: Continuous Variables

\begin{tabular}{llllll}
\hline Variables & Obs & Mean & Minimum & Maximum & Std. Dev. \\
\hline INTRST & 660 & 0.0822 & -0.0016 & 0.2109 & 0.0356 \\
FINCOV & 660 & 1.7955 & 0.0000 & 6.0000 & 1.4373 \\
GENCOV & 660 & 4.2606 & 0.0000 & 27.0000 & 3.8368 \\
LOANSIZE & 660 & 25.2016 & 18.4207 & 29.4588 & 1.9070 \\
MTRTY & 660 & 3.4016 & 0.6931 & 5.3753 & 0.9103 \\
NUMLDRS & 660 & 0.4939 & 0.0000 & 21.0000 & 1.5670 \\
TANGIBILITY & 660 & 0.3826 & 0.0018 & 0.8910 & 0.2494 \\
SIZE & 660 & 29.0618 & 26.3958 & 31.4516 & 1.3172 \\
MTB & 660 & 2.4439 & 0.3850 & 8.7900 & 2.2401 \\
ABACR & 660 & 0.0726 & 0.0004 & 0.2404 & 0.0611 \\
LEV & 660 & 0.1839 & 0.0000 & 0.5847 & 0.1489 \\
PROF & 660 & 0.1005 & -0.0458 & 0.3000 & 0.0684 \\
CFVOL & 660 & 0.0319 & 0.0101 & 0.0654 & 0.0179 \\
\hline
\end{tabular}


Panel B: Indicator Variables

\begin{tabular}{lll}
\hline Variables & Dummy $=1$ & Dummy = \\
MAO & $47.27 \%$ & $52.73 \%$ \\
AFTER MAO & $56.52 \%$ & $43.48 \%$ \\
INCONSISTENCY & $37.27 \%$ & $62.73 \%$ \\
INADQCY & $10: 00 \%$ & $90.00 \%$ \\
ACCTCHG & $28.64 \%$ & $71.36 \%$ \\
RESTATEMENT & $8.64 \%$ & $91.36 \%$ \\
MU & $2.87 \%$ & $97.13 \%$ \\
GC & $7: 12 \%$ & $92.88 \%$ \\
COLLATERAL & $82.88 \%$ & $17: 12 \%$ \\
INSTITOR & $0.76 \%$ & $99.24 \%$ \\
REVOLVER & $7: 27 \%$ & $92.73 \%$ \\
\hline
\end{tabular}

Table 3. Regression Results - Interest Spread

\begin{tabular}{|c|c|c|c|c|c|c|}
\hline Variables & Coef. & $\mathrm{P}>\mathrm{t}$ & Coef. & $\mathrm{P}>\mathrm{t}$ & Coef. & $\mathrm{P}>\mathrm{t}$ \\
\hline MAO & -0.0020 & 0.2090 & & & & \\
\hline INCONSISTENCY & & & -0.0041 & $0.0865 *$ & & \\
\hline INADQCY & & & 0.0025 & 0.3525 & & \\
\hline ACCTCHG & & & & & -0.0030 & 0.1645 \\
\hline RESTATEMENT & & & & & -0.0073 & $0.0545^{*}$ \\
\hline $\mathrm{MU}$ & & & & & 0.0015 & 0.4200 \\
\hline GC & & & & & 0.0028 & 0.3640 \\
\hline AFTERMAO & 0.0060 & $0.0115 * *$ & 0.0074 & $0.0095 * * *$ & 0.0073 & $0.0095 * * *$ \\
\hline FINCOV & -0.0046 & $0.0000 * * *$ & -0.0046 & $0.0000 * * *$ & -0.0046 & $0.0000 * * *$ \\
\hline LOANSIZE & -0.0055 & $0.0000 * * *$ & -0.0055 & $0.0000 * * *$ & -0.0055 & $0.0000 * * *$ \\
\hline MTRTY & 0.0059 & $0.0000 * * *$ & 0.0059 & $0.0000 * * *$ & 0.0060 & $0.0000 * * *$ \\
\hline COLLATERAL & 0.0078 & $0.0145 * *$ & 0.0076 & $0.0150 * *$ & 0.0076 & $0.0150 * *$ \\
\hline NUMLDRS & -0.0020 & $0.0005^{* * *}$ & -0.0021 & $0.0005 * * *$ & -0.0021 & $0.0005 * * *$ \\
\hline INSTITOR & 0.0218 & $0.0005 * * *$ & 0.0226 & $0.0000 * * *$ & 0.0243 & $0.0000 * * *$ \\
\hline REVOLVER & 0.0145 & $0.0025 * * *$ & 0.0150 & $0.0020 * * *$ & 0.0150 & $0.0020 * * *$ \\
\hline SIZE & -0.0013 & 0.1570 & -0.0013 & 0.1535 & -0.0013 & 0.1510 \\
\hline LEV & -0.0281 & $0.0010 * * *$ & -0.0279 & $0.0010 * * *$ & -0.0273 & $0.0015 * * *$ \\
\hline PROF & -0.0683 & $0.0010 * * *$ & -0.0628 & $0.0035 * * *$ & -0.0625 & $0.0050 * * *$ \\
\hline TANGIBILITY & -0.0067 & $0.0940 *$ & -0.0078 & $0.0565^{*}$ & -0.0081 & $0.0475^{* *}$ \\
\hline MTB & 0.0020 & $0.0010 * * *$ & 0.0019 & $0.0030 * * *$ & 0.0019 & $0.0035 * * *$ \\
\hline CFVOL & -0.2870 & $0.0005 * * *$ & -0.2841 & $0.0005 * * *$ & -0.2720 & $0.0010 * * *$ \\
\hline ABACR & -0.0501 & $0.0105 * *$ & -0.0532 & $0.0090 * * *$ & -0.0529 & $0.0095 * * *$ \\
\hline D2014 & 0.0009 & 0.3900 & -0.0004 & 0.4570 & 0.0001 & 0.4920 \\
\hline D2015 & 0.0091 & $0.0065 * * *$ & 0.0084 & $0.0160 * *$ & 0.0085 & $0.0140 * *$ \\
\hline D2016 & 0.0029 & 0.1770 & 0.0030 & 0.1655 & 0.0030 & 0.1675 \\
\hline CONS & 0.2565 & 0.0000 & 0.2570 & 0.0000 & 0.2563 & 0.0000 \\
\hline Prob>f & \multicolumn{2}{|r|}{0.0000} & \multicolumn{2}{|r|}{0.0000} & \multicolumn{2}{|c|}{0.0000} \\
\hline R-squared & \multicolumn{2}{|r|}{0.3410} & \multicolumn{2}{|r|}{0.3426} & \multicolumn{2}{|c|}{0.3435} \\
\hline
\end{tabular}

Table 4. Regression Results - Financial Covenant

\begin{tabular}{|c|c|c|c|c|c|c|}
\hline Variables & Coef. & $\mathrm{P}>\mathrm{t}$ & Coef. & $\mathrm{P}>\mathrm{t}$ & Coef. & $\mathrm{P}>\mathrm{t}$ \\
\hline MAO & -0.1817 & $0.0525^{*}$ & & & & \\
\hline INCONSISTENCY & & & -0.1123 & 0.1980 & & \\
\hline INADQCY & & & -0.3346 & $0.0410 * *$ & & \\
\hline АCCTCHG & & & & & -0.1421 & 0.1585 \\
\hline RESTATEMENT & & & & & -0.0105 & 0.4795 \\
\hline $\mathrm{MU}$ & & & & & -0.6477 & $0.0205 * *$ \\
\hline GC & & & & & -0.1955 & 0.1890 \\
\hline AFTERMAO & 0.2839 & $0.008 * * *$ & 0.2370 & $0.0310 * *$ & 0.2314 & 0.0345 \\
\hline INTRST & -8.8501 & 0.0000 & -8.7526 & $0.0000 * * *$ & -8.6993 & $0.0000 *$ \\
\hline LOANSIZE & 0.0208 & 0.2555 & 0.0217 & 0.2470 & 0.0228 & 0.2360 \\
\hline
\end{tabular}




\begin{tabular}{|c|c|c|c|c|c|c|}
\hline MTRTY & -0.0071 & 0.4555 & -0.0096 & 0.4405 & -0.0072 & 0.4550 \\
\hline COLLATERAL & 0.2545 & $0.0400 * *$ & 0.2599 & $0.0370^{* *}$ & 0.2616 & $0.0360 * *$ \\
\hline NUMLDRS & 0.0841 & $0.0015 * * *$ & 0.0855 & $0.0015 * * *$ & 0.0886 & $0.0010 * * *$ \\
\hline INSTITOR & 0.9939 & $0.0440 * *$ & 0.9643 & $0.0495 * *$ & 0.8997 & $0.0635 *$ \\
\hline REVOLVER & -0.0283 & 0.4445 & -0.0460 & 0.4105 & -0.0471 & 0.4085 \\
\hline SIZE & 0.2024 & $0.0000 * * *$ & 0.2022 & $0.0000 *$ & 0.1993 & $0.0000 *$ \\
\hline LEV & 0.6866 & $0.0415^{* *}$ & 0.6811 & $0.0425 * *$ & 0.7262 & $0.0345 * *$ \\
\hline PROF & 3.4577 & $0.0000 * * *$ & 3.2715 & $0.0000 *$ & 3.4493 & $0.0000 *$ \\
\hline TANGIBILITY & 0.2785 & 0.1010 & 0.3155 & $0.0775 *$ & 0.3093 & $0.0825 *$ \\
\hline MTB & -0.0337 & $0.0860 *$ & -0.0292 & 0.1225 & -0.0341 & $0.089 *$ \\
\hline CFVOL & -3.6472 & 0.1430 & -3.7156 & 0.1385 & -4.3177 & 0.1070 \\
\hline ABACR & -1.3585 & $0.0565 *$ & -1.2449 & $0.0755^{*}$ & -1.2429 & $0.0755 *$ \\
\hline D2014 & -0.2099 & $0.0595 *$ & -0.1671 & 0.1190 & -0.1762 & 0.1095 \\
\hline D2015 & 0.1252 & 0.2155 & 0.1487 & 0.1775 & 0.1627 & 0.1565 \\
\hline D2016 & 0.0219 & 0.4410 & 0.0189 & 0.4490 & 0.0199 & 0.4465 \\
\hline Cons & -4.4479 & 0.0015 & -4.4770 & 0.0010 & -4.4284 & 0.0015 \\
\hline Prob>f & \multicolumn{2}{|c|}{0.0000} & \multicolumn{2}{|c|}{0.0000} & \multicolumn{2}{|c|}{0.0000} \\
\hline R-squared & \multicolumn{2}{|c|}{0.2280} & \multicolumn{2}{|c|}{0.2296} & \multicolumn{2}{|c|}{0.2316} \\
\hline
\end{tabular}

Table 5. Regression Results - General Covenant

\begin{tabular}{|c|c|c|c|c|c|c|}
\hline Variables & Coef. & $\mathrm{P}>\mathrm{t}$ & Coef. & $\mathrm{P}>\mathrm{t}$ & Coef. & $P>t$ \\
\hline MAO & -0.2539 & 0.2320 & & & & \\
\hline INCONSISTENCY & & & -0.7391 & $0.0270 * *$ & & \\
\hline INADQCY & & & 0.8165 & 0.1310 & & \\
\hline ACCTCHG & & & & & -0.6746 & $0.0490 * *$ \\
\hline RESTATEMENT & & & & & -1.0554 & $0.0270 * *$ \\
\hline MU & & & & & 3.8297 & $0.0010 * * *$ \\
\hline GC & & & & & -0.5150 & 0.2545 \\
\hline AFTERMAO & -0.1327 & 0.3675 & 0.1958 & 0.3235 & 0.2529 & 0.2780 \\
\hline INTRST & 21.6407 & $0.0000 * * *$ & 20.9583 & $0.0000 *$ & 20.7583 & $0.0000 *$ \\
\hline LOANSIZE & 0.1285 & $0.0665 *$ & 0.1224 & $0.0735 *$ & 0.1125 & $0.0865^{*}$ \\
\hline MTRTY & 0.2052 & 0.1315 & 0.2226 & 0.1115 & 0.1906 & 0.1465 \\
\hline COLLATERAL & -0.3742 & 0.2085 & -0.4116 & 0.1855 & -0.4315 & 0.1755 \\
\hline NUMLDRS & -0.0384 & 0.3235 & -0.0486 & 0.2690 & -0.0746 & 0.1720 \\
\hline INSTITOR & -0.2311 & 0.4200 & -0.0242 & 0.4915 & 0.2279 & 0.4270 \\
\hline REVOLVER & 0.5788 & 0.1055 & 0.7024 & $0.0640 *$ & 0.7049 & $0.0670 *$ \\
\hline SIZE & -0.5356 & $0.0000 * * *$ & -0.5342 & $0.0000 * * *$ & -0.5067 & $0.0005 * * *$ \\
\hline LEV & -0.5269 & 0.2915 & -0.4886 & 0.3040 & -1.0020 & 0.1385 \\
\hline PROF & -7.5090 & $0.0025 * * *$ & -6.2059 & $0.0270 * *$ & -7.8727 & $0.0030 * * *$ \\
\hline TANGIBILITY & 1.1002 & $0.0420 * *$ & 0.8412 & $0.0915 *$ & 0.9585 & $0.0625^{*}$ \\
\hline MTB & 0.0342 & 0.3335 & 0.0027 & 0.4865 & 0.0422 & 0.2995 \\
\hline CFVOL & 3.0340 & 0.3880 & 3.5125 & 0.3705 & 6.7781 & 0.2660 \\
\hline $\mathrm{ABACR}$ & 2.7189 & 0.1385 & 1.9235 & 0.2215 & 1.8639 & 0.2280 \\
\hline D2014 & 0.3140 & 0.2130 & 0.0144 & 0.4865 & 0.0096 & 0.4915 \\
\hline D2015 & -0.7798 & $0.0345 * *$ & -0.9442 & $0.0160 * *$ & -1.0956 & $0.0065^{* *}$ \\
\hline D2016 & 0.1869 & 0.3305 & 0.2082 & 0.6230 & 0.2007 & 0.3145 \\
\hline Cons & 14.6670 & 0.0005 & 14.8706 & 0.0010 & 14.5075 & 0.0005 \\
\hline Prob>f & \multicolumn{2}{|c|}{0.0000} & \multicolumn{2}{|c|}{0.0000} & \multicolumn{2}{|c|}{0.0000} \\
\hline R-squared & \multicolumn{2}{|c|}{0.1242} & \multicolumn{2}{|c|}{0.1322} & \multicolumn{2}{|c|}{0.1570} \\
\hline
\end{tabular}

Table 6. Regression Results - Loan Size

\begin{tabular}{|c|c|c|c|c|c|c|}
\hline Variables & Coef. & $\mathrm{P}>\mathrm{t}$ & Coef. & $\mathrm{P}>\mathrm{t}$ & Coef. & $P>t$ \\
\hline MAO & -0.0682 & 0.3130 & & & & \\
\hline INCONSISTENCY & & & -0.1324 & 0.2115 & & \\
\hline INADQCY & & & 0.0744 & 0.3785 & & \\
\hline ACCTCHG & & & & & -0.1194 & 0.2505 \\
\hline RESTATEMENT & & & & & -0.1785 & 0.2400 \\
\hline
\end{tabular}




\begin{tabular}{|c|c|c|c|c|c|c|}
\hline $\mathrm{MU}$ & & & & & 0.2848 & 0.2365 \\
\hline GC & & & & & -0.0180 & 0.4740 \\
\hline AFTERMAO & 0.1159 & 0.2160 & 0.1591 & 0.1585 & 0.1624 & 0.1540 \\
\hline INTRST & -16.2659 & $0.0000 * * *$ & -16.3306 & $0.0000 * * *$ & -16.3268 & $0.0000 * * *$ \\
\hline FINCOV & 0.0324 & 0.2555 & 0.0338 & 0.2470 & 0.0357 & 0.2360 \\
\hline MTRTY & 0.3055 & $0.0000 * * *$ & 0.3076 & $0.0000 * * *$ & 0.3055 & $0.0000 * * *$ \\
\hline COLLATERAL & -0.0796 & 0.3305 & -0.0848 & 0.3200 & -0.0866 & 0.3170 \\
\hline NUMLDRS & 0.1764 & $0.0000 *$ & 0.1748 & $0.0000 * * *$ & 0.1726 & $0.0000 * * *$ \\
\hline INSTITOR & 0.7905 & 0.1390 & 0.8159 & 0.1315 & 0.8446 & 0.1260 \\
\hline REVOLVER & 0.5848 & $0.0105 * *$ & 0.6007 & $0.0090 * * *$ & 0.6009 & $0.0090 * * *$ \\
\hline SIZE & 0.3441 & $0.0000 * * *$ & 0.3437 & $0.0000 * * *$ & 0.3449 & $0.0000 * * *$ \\
\hline LEV & 0.3838 & 0.1095 & 0.3876 & 0.2165 & 0.3532 & 0.2395 \\
\hline PROF & -3.0394 & $0.0025 * * *$ & -2.8685 & $0.0050 * * *$ & -2.9901 & $0.0040 * * *$ \\
\hline TANGIBILITY & -0.6643 & $0.0075^{* * *} *$ & -0.6986 & $0.0060 * * *$ & -0.6926 & $0.0065^{* * *} *$ \\
\hline MTB & 0.0011 & 0.4855 & -0.0030 & 0.4615 & 0.0001 & 0.4990 \\
\hline CFVOL & 5.7004 & $0.0905^{*}$ & 5.7644 & $0.0880^{*}$ & 6.0875 & $0.0805^{*}$ \\
\hline ABACR & 1.4396 & $0.0895 *$ & 1.3346 & 0.1085 & 1.3332 & 0.1090 \\
\hline D2014 & -0.0838 & 0.3095 & -0.1233 & 0.2430 & -0.1198 & 0.2520 \\
\hline D2015 & 0.3045 & $0.0620 *$ & 0.2823 & $0.0795 *$ & 0.2718 & $0.0890 *$ \\
\hline D2016 & -0.0048 & 0.4895 & -0.0020 & 0.4955 & -0.0027 & 0.4945 \\
\hline Cons & 15.5077 & 0.0000 & 15.5280 & 0.0000 & 15.4954 & 0.0000 \\
\hline Prob>f & \multirow{2}{*}{\multicolumn{2}{|c|}{$\begin{array}{l}0.0000 \\
0.3169\end{array}$}} & \multicolumn{2}{|c|}{0.0000} & \multicolumn{2}{|c|}{0.0000} \\
\hline R-squared & & & & & 0.3 & \\
\hline
\end{tabular}

Table 7. Regression Results - Maturity

\begin{tabular}{|c|c|c|c|c|c|c|}
\hline Variables & Coef. & $P>t$ & Coef. & $\mathrm{P}>\mathrm{t}$ & Coef. & $\mathrm{P}>\mathrm{t}$ \\
\hline MAO & -0.0778 & 0.1305 & & & & \\
\hline INCONSISTENCY & & & -0.0339 & 0.3395 & & \\
\hline INADQCY & & & -0.1748 & $0.0705^{*}$ & & \\
\hline ACCTCHG & & & & & -0.0612 & 0.2425 \\
\hline RESTATEMENT & & & & & 0.0414 & 0.3700 \\
\hline MU & & & & & 0.0078 & 0.4840 \\
\hline GC & & & & & -0.2533 & $0.0320 * *$ \\
\hline AFTERMAO & 0.2220 & $0.0010 * * *$ & 0.1922 & $0.0070 * * *$ & 0.1952 & $0.0065^{* * * *}$ \\
\hline INTRST & 4.2609 & $0.0000 * * *$ & 4.3075 & $0.0000 * * *$ & 4.3339 & $0.0000 * * *$ \\
\hline FINCOV & -0.0027 & 0.4555 & -0.0037 & 0.4405 & -0.0028 & 0.4550 \\
\hline LOANSIZE & 0.0747 & $0.0000 * * *$ & 0.0752 & $0.0000 * * *$ & 0.0745 & $0.0000 * * *$ \\
\hline COLLATERAL & 0.4237 & $0.0000 * * *$ & 0.4267 & $0.0000 * * *$ & 0.4235 & $0.0000^{* * *}$ \\
\hline NUMLDRS & 0.0621 & $0.0000 * * *$ & 0.0630 & $0.0000 * * *$ & 0.0616 & $0.0000 * * *$ \\
\hline INSTITOR & -0.1154 & 0.3745 & -0.1330 & 0.3560 & -0.1707 & 0.3200 \\
\hline REVOLVER & -0.7176 & $0.0000 * * *$ & -0.7277 & $0.0000 * * *$ & -0.7263 & $0.0000 * * *$ \\
\hline SIZE & 0.0095 & 0.3745 & 0.0096 & 0.3740 & 0.0110 & 0.3560 \\
\hline LEV & 0.9012 & $0.0000 * * *$ & 0.8970 & $0.0000 * * *$ & 0.8513 & $0.0005 * * *$ \\
\hline PROF & 0.9752 & $0.0355^{* *}$ & 0.8589 & $0.0600 *$ & 0.7588 & $0.0875^{*}$ \\
\hline TANGIBILITY & 0.3400 & $0.006 * * *$ & 0.3632 & $0.0040 * * *$ & 0.3770 & $0.0030 * * *$ \\
\hline MTB & 0.0356 & $0.0095 * * *$ & 0.0384 & $0.0065 * * *$ & 0.0395 & $0.0055^{* * *}$ \\
\hline CFVOL & -13.5936 & $0.0000 * * *$ & -13.6189 & $0.0000 * * *$ & -13.7333 & $0.0000 * * *$ \\
\hline ABACR & -0.0844 & 0.4370 & -0.0135 & 0.4900 & -0.0216 & 0.4840 \\
\hline D2014 & -0.0840 & 0.1565 & -0.0570 & 0.2575 & -0.0700 & 0.2145 \\
\hline D2015 & 0.0522 & 0.2975 & 0.0671 & 0.2495 & 0.0553 & 0.2895 \\
\hline D2016 & 0.2333 & $0.0050 * * *$ & 0.2310 & $0.0055 * * *$ & 0.2302 & $0.0055^{* * *}$ \\
\hline Cons & 0.4040 & 0.3295 & 0.3806 & 0.3390 & 0.3763 & 0.3405 \\
\hline Prob>f & \multicolumn{2}{|c|}{0.0000} & \multicolumn{2}{|c|}{0.0000} & \multicolumn{2}{|c|}{0.0000} \\
\hline R-squared & \multicolumn{2}{|c|}{0.2669} & \multicolumn{2}{|c|}{0.2680} & \multicolumn{2}{|c|}{0.2704} \\
\hline
\end{tabular}

$* * *=$ significant at $1 \% ; * *=$ significant at $5 \% ; *=$ significant at $10 \%$ 
Table 8. Regression Results - Collateral

\begin{tabular}{|c|c|c|c|c|c|c|}
\hline Variables & Coef. & $\mathrm{P}>\mathrm{z}$ & Coef. & $\mathrm{P}>\mathrm{Z}$ & Coef. & $\mathrm{P}>\mathrm{z}$ \\
\hline $\mathrm{MAO}$ & 0.1211 & 0.3265 & & & & \\
\hline INCONSISTENCY & & & -0.0456 & 0.4390 & & \\
\hline INADQCY & & & 0.7051 & 0.1010 & & \\
\hline ACCTCHG & & & & & -0.0023 & 0.4970 \\
\hline RESTATEMENT & & & & & -0.1882 & 0.3300 \\
\hline MU & & & & & 1.4312 & 0.1025 \\
\hline GC & & & & & 0.4299 & 0.2445 \\
\hline AFTERMAO & -0.9253 & $0.0005 * * *$ & -0.8038 & $0.0040 * * *$ & -0.8022 & $0.0040 * * *$ \\
\hline INTRST & 9.1139 & $0.0080 * * *$ & 8.7330 & $0.0115^{* *}$ & 9.0432 & $0.0100 *$ \\
\hline FINCOV & 0.1435 & $0.0700^{*}$ & 0.1602 & $0.0520 *$ & 0.1662 & $0.0460 * *$ \\
\hline LOANSIZE & -0.0382 & 0.3060 & -0.0450 & 0.2775 & -0.0446 & 0.2795 \\
\hline MTRTY & 0.7995 & $0.0000 * * *$ & 0.8082 & $0.0000 * * *$ & 0.8106 & $0.0000 * * *$ \\
\hline NUMLDRS & -0.0592 & 0.1655 & -0.0711 & 0.1195 & -0.0793 & $0.0950 *$ \\
\hline INSTITOR & -1.2856 & 0.1145 & -1.2085 & 0.1295 & -1.1501 & 0.1410 \\
\hline REVOLVER & -0.5783 & $0.0610^{*}$ & -0.5238 & $0.0815^{*}$ & -0.5269 & $0.0800 *$ \\
\hline SIZE & -0.6973 & $0.0000 * * *$ & -0.6991 & $0.0000 * * *$ & -0.6965 & $0.0000 * * *$ \\
\hline LEV & 1.1674 & 0.1020 & 1.2056 & $0.0955^{*}$ & 1.2224 & $0.0925^{*}$ \\
\hline PROF & -2.5787 & 0.1095 & -2.2173 & 0.1495 & -2.4988 & 0.1245 \\
\hline TANGIBILITY & 0.2557 & 0.3140 & 0.1370 & 0.3995 & 0.1926 & 0.3615 \\
\hline MTB & 0.0182 & 0.3830 & 0.0145 & 0.4080 & 0.0201 & 0.3745 \\
\hline CFVOL & 10.0826 & 0.1060 & 10.3887 & 0.1010 & 11.5130 & $0.0830 *$ \\
\hline ABACR & 1.8742 & 0.1870 & 1.6560 & 0.2185 & 1.8096 & 0.1995 \\
\hline D2014 & -0.0357 & 0.4555 & -0.1147 & 0.3630 & -0.1053 & 0.3760 \\
\hline D2015 & -0.5568 & $0.0590 *$ & -0.6058 & $0.0455^{* *}$ & -0.6485 & $0.0370 * *$ \\
\hline D2016 & -0.1346 & 0.3535 & -0.1087 & 0.3810 & -0.1227 & 0.3665 \\
\hline Cons & 19.7252 & 0.0000 & 19.9083 & 0.0000 & 19.7410 & 0.0000 \\
\hline Prob>f & \multicolumn{2}{|c|}{0.0000} & \multicolumn{2}{|c|}{0.0000} & \multicolumn{2}{|c|}{0.0000} \\
\hline R-squared & \multicolumn{2}{|c|}{0.2035} & \multicolumn{2}{|c|}{0.2063} & \multicolumn{2}{|c|}{0.2077} \\
\hline
\end{tabular}

$* * *=$ significant at $1 \% ; * *=$ significant at $5 \% ; *=$ significant at $10 \%$

These results are contrary to the study of Chen [2]. For ACCTCHG, could be due to in Indonesia there are many new accounting standards which begin effective. In 2015, there are new and amendment of accounting standard effective for the year. This resulted in the type of modified audit opinion which is not the results of an aggressive accounting policy, but due to the need to comply with the standard. These circumstances make the use of general covenants decreased due to no evidence of less credibility of the information in the financial statements.

For variable RESTATEMENT also have a negative effect on the general covenants and interest rate. This means restatements interpreted as good news. Palmrose, Richardson, and Scholz [27] , suggest that, although uncommon, the effect of the restatement of financial statements interpreted as good news if the restatement is causing an increase in revenue.

MU variables also affect general covenants positively. This means that when companies get MAO caused by a material uncertainty in the business, the amount of general covenants in the credit facility will be increased. The company controls a potential loss due to uncertainty about the company's business through general covenants[2] .

INADQCY affect the dependent variable only financial covenant and the effect is negative. MU variables that also affect financial covenant negatively. The results are in line with what was predicted that the decline in the quality of financial information reflected in the audit report, the lender will reduce the requirements of the agreement that rely on the numbers in the financial statements[2]. That is because the figures used in the financial covenants derived from the financial statements cannot be used by lenders to monitor and control the company due to the declining credibility of financial statements.

For GC variable, this variable only has negative association with loan maturity. This meant 
that when the company gets MAO caused by going concern will affect in decreasing loan maturity. This is in accordance with Diamond [28] . A company that has a high risk, in this study who received gets going-concern opinion, will have a term of the loan is lower than the company that has a low risk.

Based on above results, we can conclude that hypothesis 1 is not supported because there is no significant evidence that modified audit opinion increase the interest spread. Hypothesis 2 is also only partially supported as modified audit opinion has significant on reducing the number of financial covenants. We only find significant effect of modified audit opinion on the number of general covenants in case material uncertainty. Hypothesis 3 is also only supported for going concern opinion on loan maturity. There is no significant evidence that modified audit opinion affect the collateral and loan size in the loan contract.

The possible explanation on these results may be due to lenders in Indonesia still relies heavily on soft information, instead of hard information. According to Baas and Schrooten [29] , soft Information using relationship lending technique that is credit distribution based on belief system and relationship that has been built well between Bank and companies, so that information can be accessed more easily by bank. While for the hard information is based on: 1) Financial Statement Lending, i.e. by using financial statements, 2) Assets Based Lending i.e. by using information related to assets that are used as collateral, 3) Credit Scoring, using the available financial data assessed on the credit score.

As explained earlier that the possible cause of insignificant effect of modified audit opinion because lenders pay more attention to other data sources, namely the characteristics of the loan and the characteristics of the company. In this study, the control variables of the research model are the characteristic of the loan and the characteristics of the company.

Variable interest rates have a negative effect on the financial covenants and a positive influence on the general covenants, consistent with Costello [30]. This variable also negatively affects the loan amount, consistent with Beatty [31].The interest rate has a positive effect on the term of the loan and the need for collateral. According to Karjalainen [32], high interest rates will increase the need for collateral. The interest rate also has a positive relationship with the term of the loan, because lenders require a liquidity premium for the loan period is longer, and thus higher interest rate [10].

LOANSIZE have a positive effect on the term of the loan. This result is consistent with Graham[10] . Regarding relations with financial covenants, the results are consistent with previous studies [2] [10]. Thus the need for collateral does not replace the need for financial covenants in the credit facility[30]. The longer loan term will require collateral[33].

NUMLDRS positively associated with financial covenants, the loan amount and term of the loan, and negatively with interest rate. These results are consistent with of Chen [2]. Increasing number of lenders in the syndicated loan will increase the number of financial covenants as more and more problems asymmetric information that should be monitored[30]. The loan amount and term of the loan increases if the number of lenders are higher, while the interest rate will be lower because of the risk of the spread of credit facilities to each lender. Variable INSTITOR positive effect on interest rates, this is because these loans have higher agency cost of debt than usual credit[30].

Company size and profitability positively effects financial covenants and negative to the general covenants. This means that information about the numbers in the financial statements in a large reliable company so that financial covenants can be used as a reference in monitoring the companies[30]. Company size and profitability negatively affect the interest rate and the need for collateral and positive relationship on the loan amount, consistent with Chen [2] .This is because lenders see a large company is a company that has a low risk [34]. 
LEV has a positive relationship with the financial covenants in support of Graham[10] and positively related to the term of the loan [35][36] [37]. LEV variable is negatively related to the interest rate. According to Karjalainen [32] it can be due to the interest rate can be increased due to the level of leverage, but the company will also reduce the level of leverage in line with interest rate increases. Variable tangibility has a negative effect with the number of loans and a positive relationship with the term of the loan. Companies with low tangibility will make the loan amount obtained when the company went bankrupt low because only a company's assets that will be acquired lender[10]. This result is consistent with Graham [10] and Chen [2].

\section{Conclusion}

This study aims to examine at the effect of a modified audit opinion on the company's debt contractual arrangements. Results of this study show that in general there is limited effect of modified audit opinion on debt contract. We do not find evidence that type of modified audit opinion causes higher interest rates. Modified audit opinion has significant effect on reducing the number of financial covenants. We only find significant effect of modified audit opinion on the number of general covenants in material uncertainty case. We also only find supporting evidence on loan maturity for going concern opinion. Modified audit opinion does not affect the possibility of the need collateral and loan size in the debt contract. These results may be due to lenders relies on soft information rather than hard information.

This study has several limitations. First, we do not include variable related to auditor characteristics (such as audit tenure) that may affect lenders decision whether to incorporate audit report information in their lending decision. As lenders may rely on soft information, future studies may include variables that capture the soft information into research model.

\section{Acknowledgement}

This research is supported by PITTA research grant.

\section{References}

[1] K. Saravanakumar, C. Yu, K. Dou, M. Wang, Y. Li, and J. Chen, "Synergistic effect of Trichoderma-derived antifungal metabolites and cell wall degrading enzymes on enhanced biocontrol of Fusarium oxysporum f. sp. cucumerinum," Biol. Control, vol. 94, pp. 37-46, 2016.

[2] P. F. Chen, S. He, Z. Ma, and D. Stice, "The information role of audit opinions in debt contracting," J. Account. Econ., vol. 61, no. 1, pp. 121-144, 2016.

[3] K. Menon and D. D. Williams, "Investor reaction to going concern audit reports," Account. Rev., vol. 85, no. 6, pp. 2075-2105, 2010.

[4] A. Guiral-Contreras, J. A. Gonzalo-Angulo, and W. Rodgers, "Information content and recency effect of the audit report in loan rating decisions," Account. Financ., vol. 47, no. 2, pp. 285-304, 2007.

[5] W. Rodgers and L. W. Johnson, "Integrating credit models using accounting information with loan officers' decision processes," Account. Financ., vol. 28, no. 2, pp. 1-22, 1988.

[6] P. H. Cahyaningrum and F. Fitriany, "Pengaruh Modified Audit Opinion Terhadap Borrowing Cash Flow Dan Investment Cash Flow. Jurnal Akuntansi dan Keuangan," Indonesia, vol. 10, no. 1, pp. 80-101, 2013.

[7] M. Jensen and W. Meckling, "Theory of the firm: managerial behavior, agency costs, and ownership structure," J. financ. econ., vol. 3, pp. 305-360, 1976. 
[8] J. S. Ang, R. A. Cole, and J. W. Lin, "Agency costs and ownership structure," J. Finance, vol. 55, no. 1, pp. 81-106, 2000.

[9] P. F. Kent and L. Munro, "Differential reporting and the effect on loan evaluations: An experimental study," Account. Forum, vol. 23, no. 4, pp. 359-377, 1999.

[10] J. R. Graham, S. Li, and J. Qiu, "Corporate misreporting and bank loan contracting," J. financ. econ., vol. 89, no. 1, pp. 44-61, 2008.

[11] M. Plumlee and T. L. Yohn, "An analysis of the underlying causes attributed to restatements," Account. Horizons, vol. 24, no. 1, pp. 41-64, 2010.

[12] H. Jiang, A. Habib, and D. Zhou, "Accounting restatements and audit quality," in China. Advances in Accounting, vol. 31, no. 1, 2015, pp. 125-135.

[13] R. Ball, R. M. Bushman, and F. P. Vasvari, "The debt contracting value of accounting information and loan syndicate structure," J. Account. Res., vol. 46, no. 2, pp. 247-287, 2008.

[14] E. Devos, S. Rahman, and D. Tsang, "Debt covenants and the speed of capital structure adjustment," J. Corp. Financ., vol. 45, pp. 1-18, 2017.

[15] M. Firth, "A note on the impact of audit qualifications on lending and credit decisions," J. Bank. Financ., vol. 4, no. 3, pp. 257-267, 1980.

[16] E. M. Bamber and R. A. Stratton, "The information content of the uncertainty-modified audit report: Evidence from bank loan officers," Account. Horizons, vol. 11, no. 2, p. 1, 1997.

[17] N. Sormunen, "Bank officers' perceptions and uses of qualified audit reports," Qual. Res. Account. Manag., vol. 11, no. 3, pp. 215-237, 2014.

[18] A. Duréndez Gómez-Guillamón, "The usefulness of the audit report in investment and financing decisions," Manag. Audit. J., vol. 18, no. 6/7, pp. 549-559, 2003.

[19] Z. Lin, Y. Jiang, and Y. Xu, "Do modified audit opinions have economic consequences? Empirical evidence based on financial constraints," China J. Account. Res., vol. 4, no. 3, pp. 135-154, 2011.

[20] L. Niemi and S. Sundgren, "Are modified audit opinions related to the availability of credit? Evidence from Finnish SMEs,” Eur. Account. Rev., vol. 21, no. 4, pp. 767-796, 2012.

[21] D. W. Blackwell, T. R. Noland, and D. B. Winters, "The value of auditor assurance: Evidence from loan pricing," J. Account. Res., vol. 36, no. 1, pp. 57-70, 1998.

[22] K. Czerney, J. J. Schmidt, and A. M. Thompson, "Does auditor explanatory language in unqualified audit reports indicate increased financial misstatement risk?," Account. Rev., vol. 89, no. 6, pp. 2115-2149, 2014.

[23] J. Jones, "Earnings management during import relief investigations," J. Account. Res., vol. 29, no. 2, p. 228, 1991.

[24] K. A. Houghton, "Audit reports: Their Impact on the loan decision process and outcome: An experiment. Accounting and business," research, vol. 14, no. 53, pp. 1520, 1938.

[25] J. R. Miller and L. M. Smith, "The Effects of the level of assurance, accounting firm, capital structure, and bank size on bank lending decisions," J. Accounting, Audit. Financ., vol. 17, no. 1, pp. 51-71, 2002.

[26] D. Johnson, K. Pany, and R. White, "Audit Reports and the Loan Decision: Actions and Perceptions. Auditing," A J. Pract. Theory, vol. 3, pp. 38-51, 1938.

[27] Z. V Palmrose, V. J. Richardson, and S. Scholz, "Determinants of market reactions to restatement announcements," J. Account. Econ., vol. 37, no. 1, pp. 59-89, 2014.

[28] D. W. Diamond, "Monitoring and reputation: The choice between bank loans and 
directly placed debt," J. Polit. Econ., vol. 99, no. 4, pp. 689-721, 1991.

[29] T. Baas and M. Schrooten, "Relationship banking and SMEs: A theoretical analysis," Small Bus. Econ., vol. 27, no. 2, pp. 127-137, 2006.

[30] A. M. Costello, "The impact of financial reporting quality on debt contracting: Evidence from internal control weakness reports," J. Account. Res., vol. 49, no. 1, pp. 97-136, 2011.

[31] A. Beatty, K. Ramesh, and J. Weber, "The importance of accounting changes in debt contracts: the cost of flexibility in covenant calculations," J. Account. Econ., vol. 33, no. 2, pp. 205-227, 2002.

[32] J. Karjalainen, "Audit quality and cost of debt capital for private firms: Evidence from," Finland. Int. J. Audit., vol. 15, no. 1, pp. 88-108, 2011.

[33] W. Voordeckers and T. Steijvers, "Business collateral and personal commitments in SME lending," J. Bank. Financ., vol. 30, no. 11, pp. 3067-3086, 2006.

[34] J. Sinkey, Commercial Bank Financial Management in the Financial Services Industry. Upper Saddle River, N. J.: Prentice Hall, 1998.

[35] M. J. Barclay and C. W. Smith, "The maturity structure of corporate debt," J. Finance, vol. 50, no. 2, pp. 609-631, 1995.

[36] S. A. Johnson, "Debt maturity and the effects of growth opportunities and liquidity risk on leverage," Rev. Financ. Stud., vol. 16, no. 1, pp. 209-236, 2003.

[37] M. H. Stohs and D. C. Mauer, "The determinants of corporate debt maturity structure," J. Bus., pp. 279-312, 1996. 